\title{
Co-Occurrence of Astasia and Unilateral Asterixis Caused by Acute Mesencephalic Infarction
}

\author{
In-Uk Song Joong-Seok Kim Jae-Young An Yeong-In Kim Kwang-Soo Lee \\ Department of Neurology, College of Medicine, The Catholic University of Korea, Seoul, South Korea
}

Dear Sir,

Astasia designates motor incoordination with inability to stand and is occasionally observed in patients with conversion hysteria [1]. Organic causes of astasia have been rarely reported. Unilateral asterixis may be caused by a focal structural brain lesion including stroke involving the thalamus, parietal lobe, frontal lobe, midbrain, basal ganglia and internal capsule, although asterixis was classically associated with metabolic derangement, particularly hepatic encephalopathy, which occurs usually bilaterally [2-5]. In addition, concurrent unilateral manifestation of astasia and asterixis due to focal cerebral lesions is extremely rare [2, 4]. To our knowledge, sudden concurrent development of asterixis and astasia as a consequence of a rostral midbrain infarction has not yet been reported. We report a patient with concurrent unilateral astasia and asterixis developed due to acute cerebral infarctions involving the rostral level of the midbrain without significant metabolic disorder.

\section{Case Report}

A 70-year-old man was admitted due to sudden onset of postural instability and dysarthria. His medical record revealed a cerebral infarction in the right centrum semiovale 8 years previously but showed no neurological condition such as essential tremor or extrapyramidal disease before the present event. He also had an 8year history of hypertension and a 5-year history of diabetes mellitus, which were well controlled. However, there were no histories of hepatitis, smoking and heavy alcohol consumption. He has taken aspirin $100 \mathrm{mg}$, clinidipine $10 \mathrm{mg}$, imidapril $10 \mathrm{mg}$ and acarbose $50 \mathrm{mg}$ daily. At the emergency room, the blood pressure was $160 / 90 \mathrm{~mm} \mathrm{Hg}$, the pulse rate $78 / \mathrm{min}$, the respiration rate $22 / \mathrm{min}$ and the body temperature $36.8^{\circ} \mathrm{C}$. On neurologic examination, he was alert and oriented with fluent but mildly slurring speech. Cranial nerve functions and muscle strength were normal with the exception of minimal drift of the right upper extremity (Medical Research Council grade IV+/V). Sensory examination revealed normal findings. The reflexes were symmetrical, and the plantar responses were flexor. When both his hands were outstretched, postural lapses with arrhythmic losses of extensor muscle tone that developed upon instruction to maintain the wrist in an extended position, so-called asterixis, or flapping tremor, was observed in the right hand. Fingerto-nose test, rapid alternation movement and heel-to-shin test were all normal. On attempt to stand, however, he showed postural instability and gait disturbance unassisted due to a tendency to tilt backward or toward right, while his ability to sit was relatively preserved. Laboratory studies, including a complete blood cell and platelet count, the erythrocyte sedimentation rate, blood electrolytes, creatinine, liver enzymes, cholesterol, triglycerides and the prothrombin and partial thromboplastin time were all normal. Magnetic resonance images of the brain on the admission day revealed high signal intensities on a diffusion weighted image and low signal intensities on an apparent diffusion coefficient map in the left rostral paramedian mesencephalon region (fig. 1). One day later, we performed surface electromyographic (EMG) recording to verify unilateral postural lapses of the right upper extremity. Surface EMG recording from right wrist extensor muscle showed an irregular brief pause of muscle activities with a duration of approximately 30-50 msec, which indicated asterixis (fig. 2). Waking and sleep electroencephalography performed to exclude partial seizure showed neither epileptic discharges nor other abnormal slowing waves. On the 4 th hospital day, asterixis disappeared, while the tendency to fall to the right posterolateral side was resolved on the 5th hospital day.

\section{Discussion}

The term astasia is defined as motor incoordiation with inability to stand despite good motor strength. The disturbance differs from cerebellar ataxia in that gait is not broad-based or lurching. It resembles the marked balance impairment of patients with vestibulocerebellar disease [4]. The responsible anatomical region for astasia or astasia-abasia has been known to be related to the thalamic lesion affecting mainly the medial ventrolateral nucleus of the thalamus because the fastigial fibers of

\section{KARGER}

Fax +41613061234 E-Mail karger@karger.ch www.karger.com
Dr. Kwang-Soo Lee

Department of Neurology, Kangnam St. Mary's Hospital

505 Banpo-dong, Seocho-gu

Seoul, 137-701 (South Korea)

Tel. +82 2590 2091, Fax +82 2599 9686, E-Mail ks1007@catholic.ac.kr 

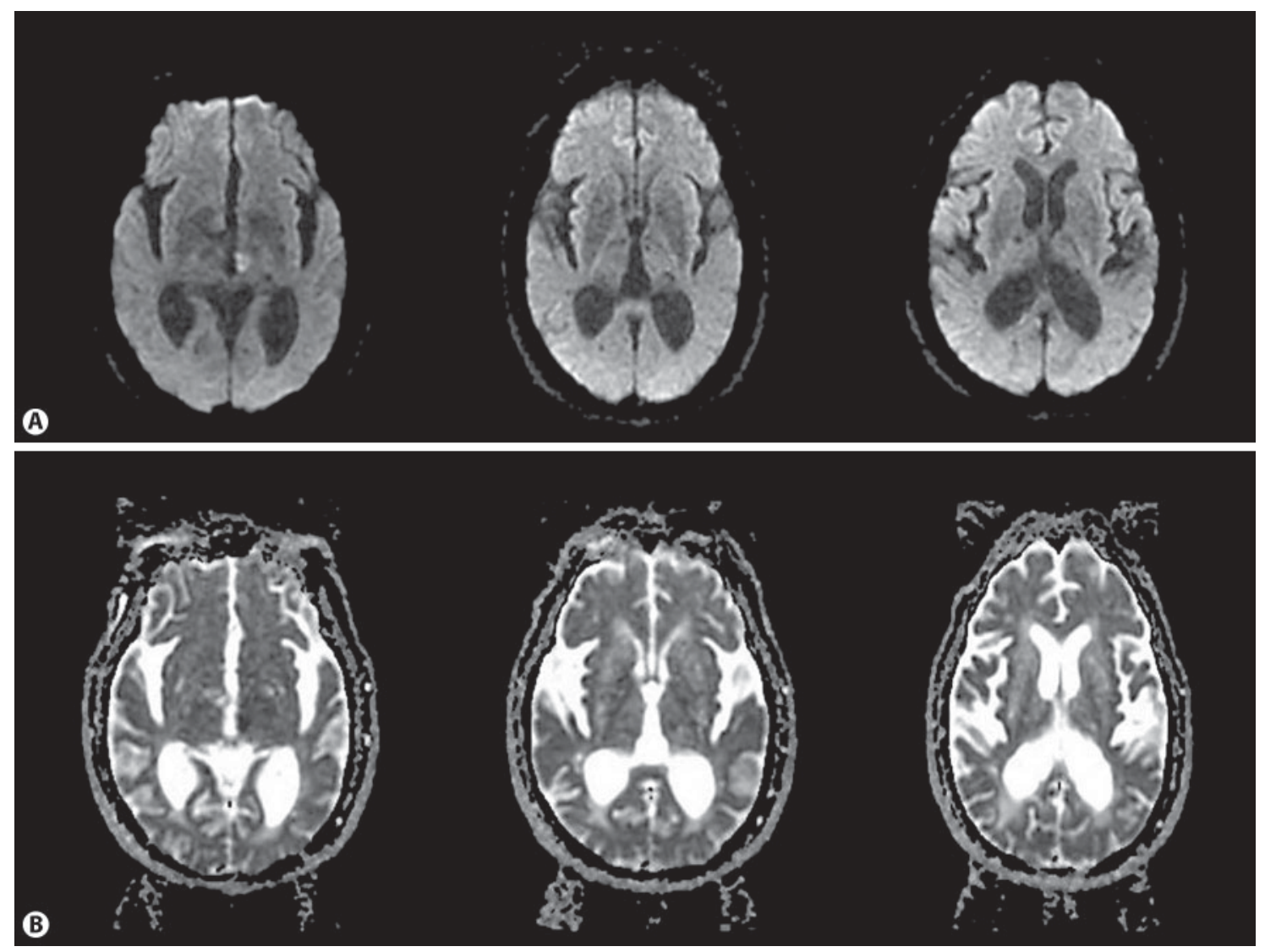

Fig. 1. Magnetic resonance images of the brain on admission day. A Diffusion weighted imaging of brain shows small and discrete high signal intensities only in the left rostral paramedian mesencephalon without lesion of other areas including thalamus. B Apparent diffuse coefficient map shows low signal intensities at comparable site.

Fig. 2. Surface electromyographic recording from right wrist extensor shows irregular brief pause of muscle activities.

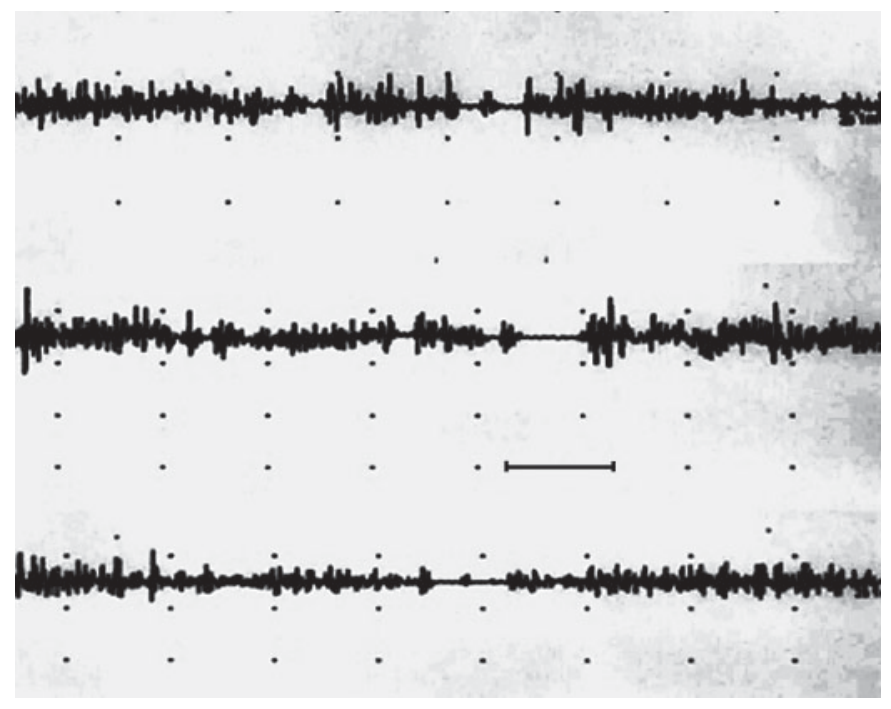


the vestibulocerebellar pathway project to the medial ventrolateral nucleus, and disruption of this pathway may cause thalamic astasia [6]. Inability to walk despite good motor strength was also reported in a patient with a red nucleus infarction involving the ascending fibers of the crossed dentatorubrothalamic tract, in a patient with a small medial capsular hemorrhage extending to the lateral portion of the ventrolateral nucleus of the thalamus and in a patient with a hemorrhage at the pontomesencephalic junction (midbrain tegmental lesion) involving the pedunculopontine area [7-9]. According to their previous report, Masdeu and Gorelick [1] also asserted that the propensity of a thalamic lesion to cause impairment of balance and gait might be because of a disconnection from vestibulocerebellar information to the vestibular cortex. However, the exact anatomical origin of atasia is not yet known.

Asterixis, one type of negative myoclonus, results from a sudden cessation of electrical activity in the extension of the limb [5]. Asterixis may occur after focal cerebral lesions involving the midbrain, thalamus, parietal lobe or the frontral cortex $[2,3,10,11]$. The pathogenic mechanism for asterixis remains elusive. Previous authors suggested that asterixis is a negative myoclonus that arose as a result of damage to a neuronal circuit sustaining postural muscle contraction [10]. This postural stability or tonic control of the ex- tremities is related to multiple brainstemspinal pathways such as the vestibulospinal, reticulospinal or rubrospinal tracts. These systems are in turn regulated by supratentorial structures; the ventrolateral nucleus of the thalamus is the area in which cerebellar-rubral or vestibulocerebellar fibers converge and is also heavily connected with the prefrontal area [3]. Since astasia and asterixis are considered to be manifestations of disordered postural or tonic balance regulated by the fastigial-thalamocortical pathway, we could explain that the lesions involving this circuit may produce these symptoms at the same time.

Our patient showed transient co-occurrence of astasia and contralateral asterixis due to acute left rostral paramedian mesencephalic infarction. The paucity or absence of motor weakness and sensory dysfunction illustrated that the corticospinal and spinothalamic tracts were generally spared. Therefore, concurrent manifestation of astasia and unilateral asterixis in our patient could be explained by disruption of cerebellar-rubral or vestibulocerebellar fiber, which projected from the fastigial fiber to the ventrolateral nucleus of the thalamus, at the rostral midbrain or midbrain-diencephalon junction level. In conclusion, we suggest that clinicians must remember to develop focal asterixis and astasia by disconnection of projection from the fastigial fiber to the ventrolateral nucleus of thalamus.
References

1 Masdeu JC, Gorelick PB: Thalamic astasia: inability to stand after unilateral thalamic lesions. Ann Neurol 1988;23:596-603.

2 Nighoghossian N, Trouillas P, Vial C, Fromet JC: Unilateral upper limb asterixis related to primary motor cortex infarction. Stroke 1995;26:326-328.

3 Kim JS: Asterixis after unilateral stroke: lesion location of 30 patients. Neurology 2001; 56:533-536.

4 Lee PH, Lee JH, Joo US: Thalamic infarct presenting with thalamic astasia. Eur J Neurol 2005;12:317-319.

5 Kim JS: Involuntary movements after anterior cerebral artery territory infarction. Stroke 2001;32:258-261.

6 Kataoka H, Sugie K, Kohara N, Ueno S: Nevel representation of astasia associated with posterior cingulate infarction. Stroke 2006; 37:e3-e5.

7 Groothuis DR, Duncan GW, Fisher CM: The human thalamocortical sensory path in the internal capsule: evidence from a small capsular hemorrhage causing a pure sensory stroke. Ann Neurol 1977;2:328-333.

8 Felice KJ, Keilson GR, Schwartz WJ: Rubral gait ataxia. Neurology 1990;40:1004-1005.

9 Masdeu JC, Alampur U, Cavaliere R, Tavoulareas G: Astasia and gait failure with damage of the pontomesencephalic locomotor region. Ann Neurol 1994;35:619-621.

10 Lee MS, Marsden CD: Movement disorders following lesions of the thalamus or subthalamic region. Mov Disord 1994;9:493-507.

11 Rossetti AO, Reichhart MD, Bogousslavsky J: Central Horner's syndrome with contralateral ataxic hemiparesis: a diencephalic alternate syndrome. Neurology 2003;61:334338. 